\title{
Using the Reference Shrinkage Curve to Estimate the Corrected Crack Volume of a Soil Layer
}

\author{
V.Y. Chertkov* \\ Agricultural Engineering Division, Faculty of Civil and Environmental Engineering, Technion, Haifa 32000, Israel
}

\begin{abstract}
To estimate the correct soil crack volume based on the shrinkage geometry factor, in addition to measurements with core samples, one also needs to know the shrinkage curve of the soil matrix without cracks. For such a curve one can use the soil reference shrinkage curve that is only stipulated by intraaggregate clay shrinkage without interaggregate cracking. The objective of this work is to illustrate such use of the reference shrinkage curve and, in addition, to argue the necessity to correct the usual estimates of the shrinkage geometry factor in order to correctly estimate the soil crack volume. The illustration is based on available data and reference shrinkage curves for eight soils.
\end{abstract}

\section{INTRODUCTION}

Knowledge of soil crack volume is important for many problems of soil hydrology. Bronswijk [1-4] suggested the estimation of the vertical-crack volume developing in a soil, based on his concept of the shrinkage geometry factor, $r_{\mathrm{s}}$. He was interested in field condition applications; that is, in the crack volume that develops in an infinite soil layer, but not in a sample. A corresponding known exact relation (neglecting horizontal cracks),

$(1-\Delta V / V)=(1-\Delta z / z)^{r_{\mathrm{S}}}$

links the variation $\Delta V$ of an initial matrix volume, $V$ in a soil layer with the variation $\Delta z$ of an initial layer thickness, $z$ at drying. The layer includes cracks along with soil matrix. The shrinkage geometry factor, $r_{\mathrm{s}}$ just determines partition of the matrix volume variation between cracks and layer thickness variation. Thus, the layer cracks enter Eq. (1) implicitly through the $r_{\mathrm{s}}$ value.

Usually for practical purposes, one deals with core sample measurements [5] instead of layer measurements. It is this replacement of a layer with a core (with coincidence between initial layer thickness, $z$ and initial core height, $z_{\mathrm{c}}$ ) that leads to the replacement of $\Delta V / V$ and $\Delta z$ in Eq. (1) with other values and that can lead to serious inaccuracy in the $r_{\mathrm{s}}$ estimates based on initially exact Eq. (1) $[6,7]$. To obtain the correct $r_{\mathrm{s}}$ values and correct crack volume estimates, in addition to measurements with cores, one also needs to know the shrinkage curve $\bar{V}(W)$ of the soil matrix without cracks [6, 7]. In general, $\bar{V}(W)$ can be measured on sufficiently small samples and at sufficiently slow drying (for an example of such use of the measured aggregate shrinkage curve from [8], see [7]). However, the use of a physically modeled soil matrix shrinkage curve $\bar{V}(W)$ is the most convenient for estimating the corrected shrinkage geometry factor and crack

*Address correspondence to this author at the Faculty of Civil and Environmental Engineering, Technion, Haifa 32000, Israel; Tel: 972-4-8292499; Fax: 972-4-829-5696; E-mail: agvictor@tx.technion.ac.il volume. In the case of pure clay one can use the model [9, 10] of a clay matrix shrinkage curve to estimate $\bar{V}(W)$ (for an example of such use, see [6, 7]). Recently, Chertkov [1113] suggested a model of the reference shrinkage curve. The model permits one to predict, for an aggregated soil with any clay content, its shrinkage curve which is only stipulated by clay shrinkage inside a soil matrix without cracking. That is, the reference shrinkage curve gives the soil matrix shrinkage curve, $\bar{V}(W)$. The objective of this work is to illustrate the application of the soil reference shrinkage curve for estimating the corrected shrinkage geometry factor and soil crack volume using available data and results from [6, 7, 11-13]. In line with that we additionally argue and illustrate the necessity of correcting the shrinkage geometry factor and crack volume that are determined from Bronswijk's approximation [5].

\section{THEORETICAL BACKGROUND}

In connection with $\Delta V / V$ it is worth reiterating that, by definition, in exact Eq. (1) the $V$ and $\Delta V$ values only relate to the layer matrix. According to [5], in practice one uses in Eq. (1), instead of the $\Delta V / V$ ratio for the soil matrix in the layer, the corresponding ratio for the relative volume variation of a core, $\Delta V_{\mathrm{c}} / V_{\mathrm{c}}$. At a given water content the $\Delta V / V$ ratio for the soil matrix in the layer coincides with a similar ratio for the soil matrix in the core, but not with $\Delta V_{\mathrm{c}} / V_{\mathrm{c}}$. Indeed, the initial state of the core is usually identified with that of the water saturation, and one considers that initial cracks are lacking. Then, the initial core volume, $V_{\mathrm{c}}$ (as $V$ ) only includes the soil matrix. However, variation of the core volume, $\Delta V_{\mathrm{c}}$ at drying (unlike $\Delta V$ ) mostly includes a crack contribution along with a soil matrix contribution. For this reason $\Delta V_{\mathrm{c}} / V_{\mathrm{c}}$ differs from $\Delta V / V$. This difference increases with drying, introducing an essential inaccuracy in the $r_{\mathrm{s}}$ value $[6,7]$.

Moreover, according to [5], in practice one can use in Eq. (1), instead of the thickness variation, $\Delta z$ of an unlimited layer, the corresponding variation of the core height, $\Delta z_{\mathrm{c}}$ (at 
$\left.z=z_{\mathrm{c}}\right)$. However, at a given water content $\Delta z_{\mathrm{c}}$ differs from $\Delta z$ $\left(\Delta z_{\mathrm{c}}<\Delta z\right)$. This difference increases with drying, introducing an additional essential inaccuracy in the $r_{\mathrm{s}}$ value $[6,7]$. The two above inaccuracies, in part mutually compensate each other. Nevertheless, the resulting $r_{\mathrm{s}}(W)$ dependence (after replacements in Eq. (1): $\Delta V / V \rightarrow \Delta V_{\mathrm{c}} / V_{\mathrm{c}}$ and $\Delta z \rightarrow \Delta z_{\mathrm{c}}$ ) is essentially distorted compared to the true one [7].

In addition to the above application of Eq. (1) (with indicated replacements and inaccuracies) for description of a situation with layer drying [1-4], there is also another application. Equation (1) in formally the same view is also the exact one for core shrinkage and cracking. In this case $\Delta z=\Delta z_{\mathrm{c}}$ (and $z=z_{\mathrm{c}}$ ), but the $V$ and $\Delta V$ values again, by definition, only relate to the core matrix, and consequently again $\Delta V / V \neq \Delta V_{\mathrm{c}} / V_{\mathrm{c}}$ because $\Delta V_{\mathrm{c}}$ can contain both crack and matrix contributions. For this reason the use in Eq. (1) for core shrinkage of $\Delta V_{\mathrm{c}} / V_{\mathrm{c}}$ instead of $\Delta V / V$ leads to inaccuracy in the $r_{\mathrm{s}}$ value $[6,7]$. Note that the $r_{\mathrm{s}}$ value in Bronswijk's approximation [5] is the same for core and layer. However, the true $r_{\mathrm{s}}$ value for the core differs from the true $r_{\mathrm{s}}$ value for the layer [7] and has another meaning. $r_{\mathrm{s}}$ determines partition of the volume variation of the core matrix between core vertical shrinkage, core lateral shrinkage, and internal cracks. Thus, core cracks enter Eq. (1) implicitly through the $r_{\mathrm{s}}$ value. Equation (1) for the core shrinkage is mostly used to characterize the shrinkage anisotropy of the core matrix. Core ma- trix shrinkage is isotropic (i.e., matrix deformations are the same for all directions) if $r_{\mathrm{s}}=3$ [1]. Available works discussing core matrix anisotropy at shrinkage (e.g., $[14,15])$ including recent ones (e.g., $[16,17])$, use $\Delta V_{\mathrm{c}} / V_{\mathrm{c}}$ for estimating $r_{\mathrm{s}}$ instead of $\Delta V / V$, ignoring the inaccuracy that is introduced in the $r_{\mathrm{s}}$ value (as a function of water content).

Chertkov [7] suggested an approach for estimating the corrected $r_{\mathrm{s}}(W)$ and corresponding corrected specific crack volume, $\bar{V}_{\mathrm{cr}}(W)$, as functions of water content $0<W<W_{\mathrm{h}}\left(W_{\mathrm{h}}\right.$ is the maximum swelling point before shrinkage starts) for both cases of layer and core. In the frame of this approach the corrected $r_{\mathrm{s}}(W)$ and $\bar{V}_{\mathrm{cr}}(W)$ are expressed through four shrinkage curves (Fig. 1): the curve ${\overline{V_{1}}}^{\prime}(W)$ of a soil layer with cracks in Bronswijk's approximation (the initial layer is composed of contacting, but disconnected cubes); the curve $\bar{V}_{1}(W)$ of a real connected soil layer with cracks; the curve $\bar{V}_{\mathrm{s}}(W)$ of a soil core sample with cracks; and the curve $\bar{V}(W)$ of a soil matrix without cracks. Given $\bar{V}_{1}(W)$, $\bar{V}_{1}^{\prime}(W), \overline{V_{\mathrm{s}}}(W)$, and $\bar{V}(W)$ (including $\bar{V}_{\mathrm{h}}=\bar{V}\left(W_{\mathrm{h}}\right)$; Fig. 1), the shrinkage geometry factor in Bronswijk's approximation, $r_{\mathrm{s}}^{\prime}\left(\Delta V / V \rightarrow \Delta V_{\mathrm{c}} / V_{\mathrm{c}}\right.$ and $\Delta z \rightarrow \Delta z_{\mathrm{c}}$; as noted above, in this

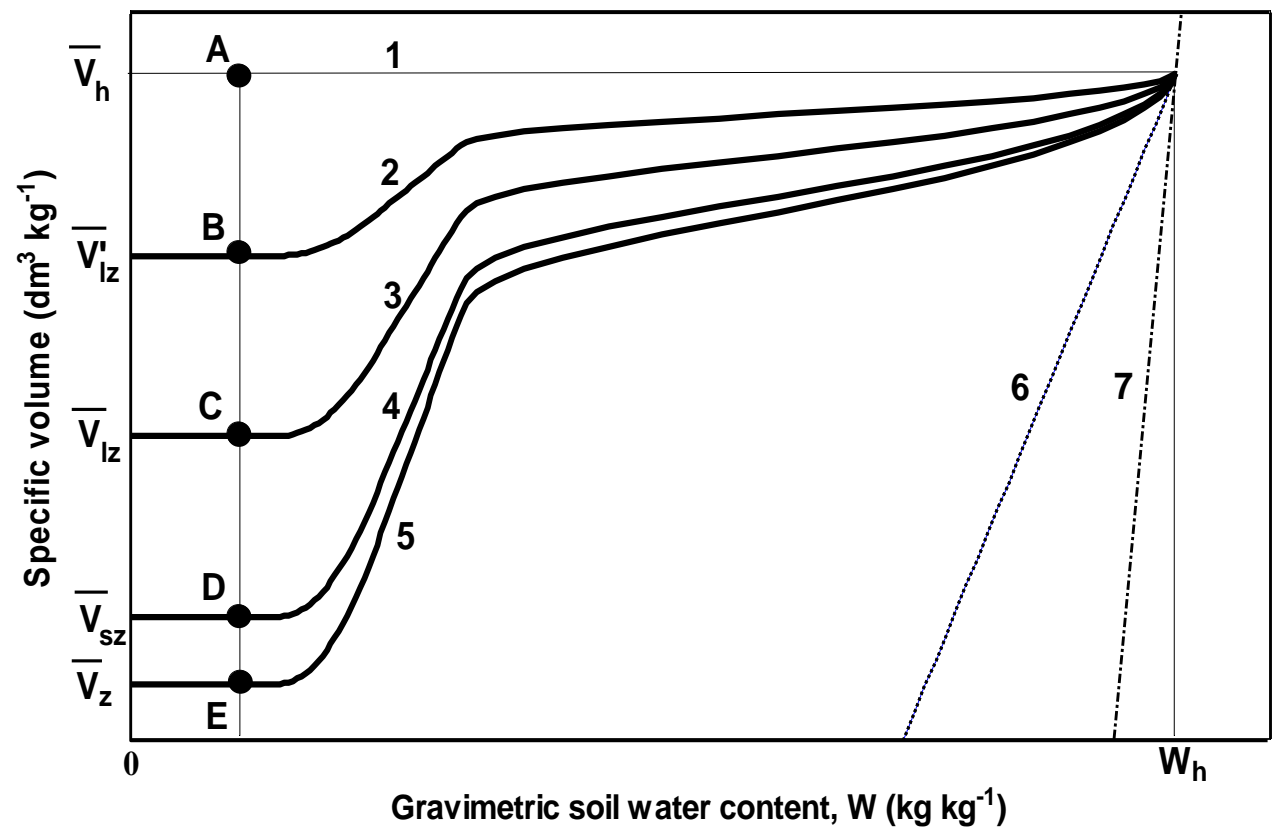

Fig. (1). Scheme of the different shrinkage curves of an aggregated soil. 1-initial specific volume (without cracks) of a shrinking and cracking soil layer. 2-shrinkage curve ${\overline{V_{1}}}^{\prime}(W)$ of a soil layer with developing cracks in Bronswijk's approximation (disconnected layer). 3corrected shrinkage curve $\overline{V_{1}}(W)$ of a (connected) soil layer with developing cracks. 4-shrinkage curve $\overline{V_{s}}(W)$ of a soil core sample with developing cracks. 5-shrinkage curve $\bar{V}(w)$ of a soil matrix without cracks (the reference shrinkage curve). 6-the line that is parallel to the reference shrinkage curve in the basic shrinkage area. 7-1:1 line (saturation or pseudo saturation line). $\bar{V}_{\mathrm{lz}}^{\prime}, \bar{V}_{\mathrm{lz}}, \bar{V}_{\mathrm{sz}}$, and $\bar{V}_{\mathrm{z}}$ designate values of corresponding specific volumes after oven drying. AB - the specific volume of layer subsidence in Bronswijk's approximation at a given $W$; AC - the corrected specific volume of layer subsidence at a given $W$; BD - the specific volume of cracks in the soil layer in Bronswijk's approximation at a given $W$; CE - the corrected specific volume of cracks in the soil layer at a given $W$; DE - the specific volume of cracks in the sample with free boundaries at a given $W$. 
approximation $r_{\mathrm{s}} \cong r_{\mathrm{s}}{ }^{\prime}$ is the same for layer and core sample) is calculated as (Fig. 1)

$r_{\mathrm{s}}^{\prime}(W)=\log \left(\bar{V}_{\mathrm{s}}(W) / \bar{V}_{\mathrm{h}}\right) / \log \left(\bar{V}_{1}^{\prime}(W) / \bar{V}_{\mathrm{h}}\right)$

the corrected shrinkage geometry factor of a soil core, $r_{\mathrm{SM}}$ is calculated as (Fig. 1)

$r_{\mathrm{sM}}(W)=\log \left(\bar{V}(W) / \bar{V}_{\mathrm{h}}\right) / \log \left(\bar{V}_{1}^{\prime}(W) / \bar{V}_{\mathrm{h}}\right)$

and the corrected shrinkage geometry factor of a soil layer, $r_{\mathrm{s}}$ is calculated as (Fig. 1)

$r_{\mathrm{s}}(W)=\log \left(\bar{V}(W) / \bar{V}_{\mathrm{h}}\right) / \log \left(\overline{V_{1}}(W) / \bar{V}_{\mathrm{h}}\right)$

The specific crack volume of the layer in Bronswijk's approximation, $\bar{V}_{\mathrm{crl}}^{\prime}(W)$ is calculated as (Fig. 1)

${\overline{V_{\mathrm{crl}}}}^{\prime}(W)={\overline{V_{1}}}^{\prime}(W)-\bar{V}_{\mathrm{s}}(W)$

The specific crack volume of the core in Bronswijk's approximation is $\bar{V}_{\mathrm{crs}}^{\prime}(W)=0$.

The corrected specific crack volume of the layer, $\bar{V}_{\mathrm{crl}}(W)$ is calculated as (Fig. 1)

$\bar{V}_{\mathrm{crl}}(W)=\overline{V_{1}}(W)-\bar{V}(W)$

and the corrected specific crack volume of the core, $\bar{V}_{\text {crs }}(W)$ is calculated as (Fig. 1)
$\bar{V}_{\mathrm{crs}}(W)=\bar{V}_{\mathrm{s}}(W)-\bar{V}(W)$

The shrinkage curves, $\bar{V}_{\mathrm{s}}(W),{\overline{V_{1}}}^{\prime}(W)$, and $\bar{V}_{1}(W)$, that, in general, contain the crack volume contribution, are found from data on vertical and lateral core shrinkage [6, 7]. The shrinkage curve, $\bar{V}(W)$ of the soil matrix without cracks is of special interest. Prediction of the reference shrinkage curve that can be used as $\bar{V}(W)$ was considered in [11-13].

\section{MATERIALS AND METHODS}

A measured shrinkage curve usually contains a crack contribution and does not coincide with the reference shrinkage curve of the soil. To estimate the reference shrinkage curve one needs to know a number of input parameters [1113]. Available data on such parameters are so far limited. Works $[11,13]$ showed that experimental shrinkage curves relating to samples from four horizons of two soils from [18] (the shrinkage of one of the soils was appreciably weaker) can be predicted as the reference shrinkage curves. That is, these soil core samples did not contain cracks at any water content. We use these eight predicted reference shrinkage curves as $\bar{V}(W)$ to illustrate the estimation of the corrected specific crack volume and corrected shrinkage geometry factor. Figs. (2 and 3) show two examples of the $\bar{V}(W)$ curve (these curves were denoted as $Y(W)$ in $[11,13])$. In addition, following [18] we assume that, for soils under consideration, the core matrix shrinkage is isotropic (we should take that into consideration because this assumption was

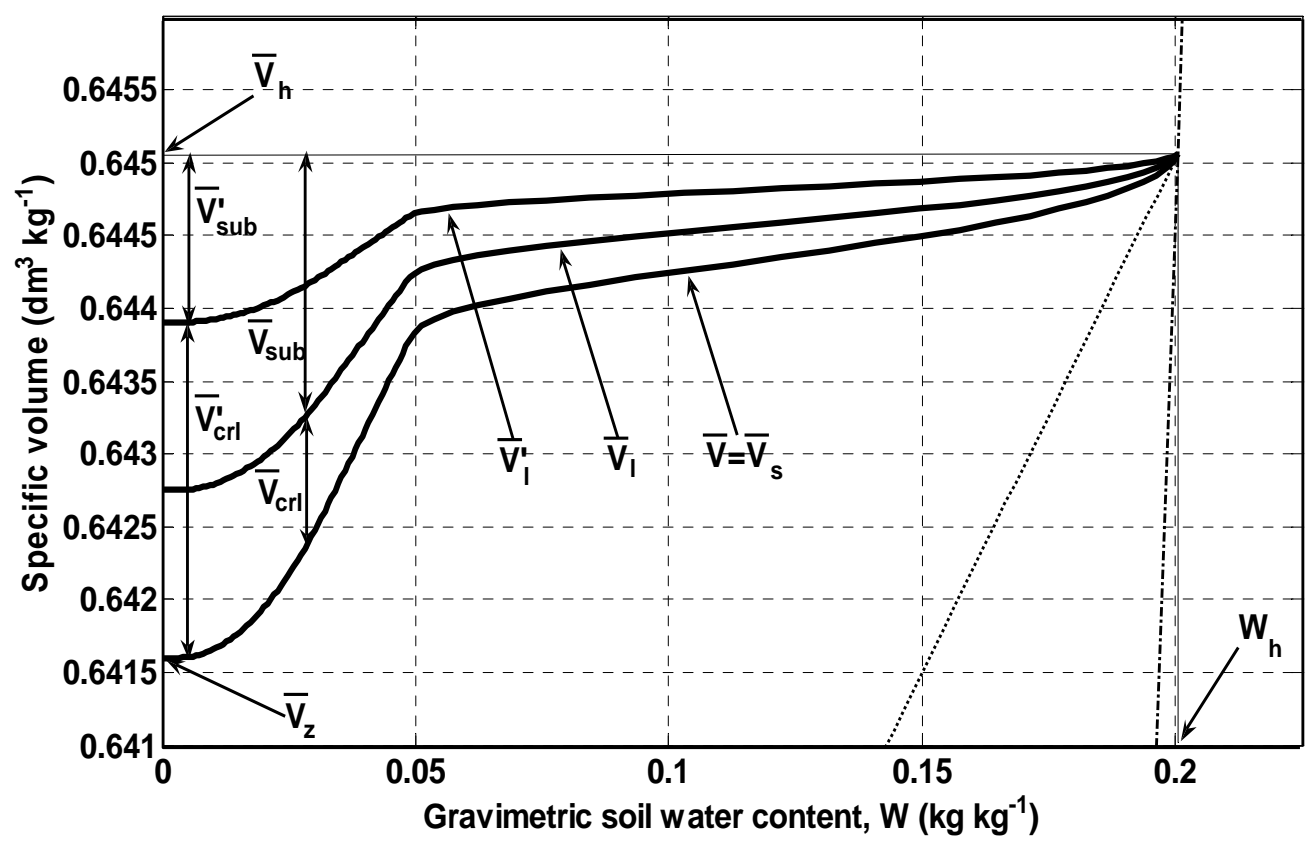

Fig. (2). The example of estimates based on the reference shrinkage curve [11, 13] for ferruginous soil, horizon A [18]. Shown are the shrinkage curves of: the soil matrix (the reference shrinkage curve), $\bar{V}(W)$; the core sample, $\bar{V}_{\mathrm{s}}(W)$ (in the case under consideration $\left.\bar{V}(W)=\bar{V}_{\mathrm{S}}(W)\right)$; and the layer before $\left(\bar{V}_{1}^{\prime}(W)\right)$ and after $\left(\bar{V}_{1}(W)\right)$ correction. The arrows show the specific volume of layer subsidence before $\left(\bar{V}_{\text {sub }}{ }^{\prime}(W)\right)$ and after $\left(\bar{V}_{\text {sub }}(W)\right)$ correction as well as the specific volume of cracks in the layer before $\left(\bar{V}_{\text {crl }}{ }^{\prime}(W)\right)$ and after $\left(\bar{V}_{\text {crl }}(W)\right)$ correction. The inclined dash-dot line is a pseudo saturation one. The dotted line is parallel to the reference shrinkage curve $(\bar{V}(W))$ in the basic shrinkage area. 


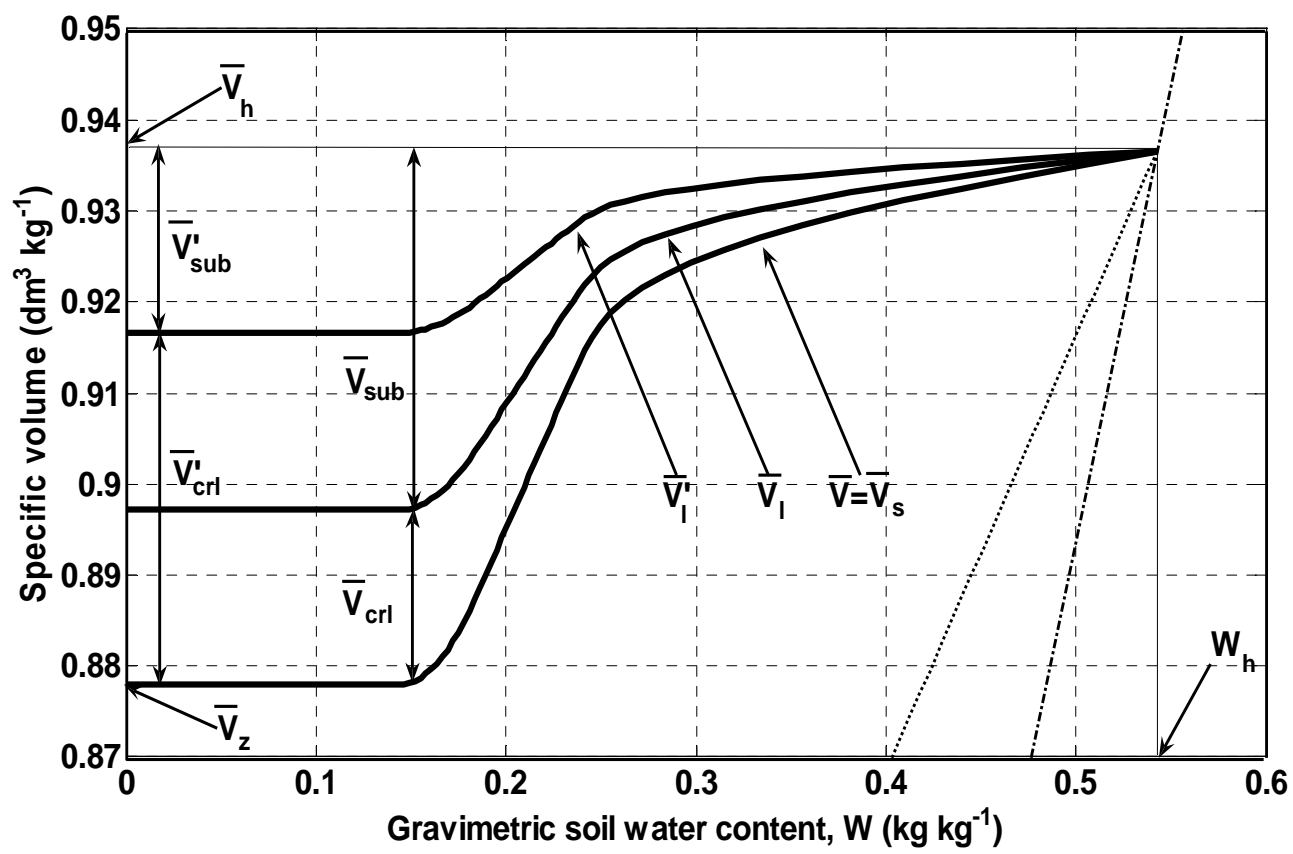

Fig. (3). The example of estimates based on the reference shrinkage curve [11, 13] for a ferralitic soil, horizon A [18]. Shown are the shrinkage curves of: the soil matrix (the reference shrinkage curve), $\bar{V}(W)$; the core sample, $\bar{V}_{\mathrm{s}}(W)$ (in the case under consideration $\bar{V}(W)=\overline{V_{\mathrm{s}}}(W)$ ); and the layer before $\left({\overline{V_{1}}}^{\prime}(W)\right)$ and after $\left(\bar{V}_{1}(W)\right)$ correction. The arrows show the specific volume of layer subsidence before $\left(\bar{V}_{\text {sub }}{ }^{\prime}(W)\right)$ and $\operatorname{after}\left(\bar{V}_{\text {sub }}(W)\right)$ correction as well as the specific volume of cracks in the layer before $\left(\bar{V}_{\text {crl }}^{\prime}(W)\right)$ and after $\left(\bar{V}_{\text {crl }}(W)\right)$ correction. The inclined dash-dot line is a pseudo saturation one. The dotted line is parallel to the reference shrinkage curve $(\bar{V}(W))$ in the basic shrinkage area.

essential for obtaining the experimental shrinkage curves). Finally, while estimating the corrected shrinkage geometry factors and crack volumes as functions of soil water content, we used the values of the core diameter, $d_{\mathrm{z}}=5.5 \mathrm{~cm}$ and height, $h_{\mathrm{z}}=3 \mathrm{~cm}$ in the oven-dried state from [18]. Thus, in the examples under consideration the core samples only contain the soil matrix. That is, (Figs. 2 and 3).

$\overline{V_{\mathrm{s}}}(W)=\bar{V}(W)$

The oven-dried mass of a sample, $m_{\mathrm{d}}$ is found to be

$m_{\mathrm{d}}=\pi d_{\mathrm{z}}^{2} h_{\mathrm{z}} /\left(4 \bar{V}_{\mathrm{z}}\right)$

where $\bar{V}_{\mathrm{z}}=\bar{V}(0)$. It follows from the isotropy of matrix shrinkage and the absence of cracks in samples that an initial core diameter, $d_{\mathrm{h}}$, current core diameter, $d(W)$, and current core height, $h(W)$ can be presented as

$d_{\mathrm{h}}=\left(\bar{V}_{\mathrm{h}} / \bar{V}_{\mathrm{z}}\right)^{1 / 3} d_{\mathrm{z}}, d(W)=\left(\bar{V}(W) / \bar{V}_{\mathrm{z}}\right)^{1 / 3} d_{\mathrm{z}}$,

and $h(W)=\left(\bar{V}(W) / \bar{V}_{\mathrm{z}}\right)^{1 / 3} h_{\mathrm{z}}$

The shrinkage curve ${\overline{V_{1}}}_{1}^{\prime}(W)$ of a soil layer with cracks in Bronswijk's approximation is found to be

${\overline{V_{1}}}^{\prime}(W)=\pi d_{\mathrm{h}}^{2} h(W) /\left(4 m_{\mathrm{d}}\right)$

The shrinkage curve $\bar{V}_{1}(W)$ of a connected soil layer with cracks is found to be [7]
$\overline{V_{1}}(W)=\bar{V}_{1}^{\prime}(W) \chi^{2}(W)$

where at the crack lack in samples

$\chi(W)=\left(1+d(W) / d_{\mathrm{h}}\right) / 2$

Then, different variants of the specific crack volume $\left(\bar{V}_{\mathrm{crl}}^{\prime}, \bar{V}_{\mathrm{crl}}\right.$, and $\left.\bar{V}_{\mathrm{crs}}\right)$ and shrinkage geometry factor $\left(r_{\mathrm{s}}^{\prime}, r_{\mathrm{sM}}\right.$, and $r_{\mathrm{s}}$ ) are found from Eq. (2)-(7).

\section{RESULTS AND DISCUSSION}

The major results for the eight soils from [18] are presented in Fig. (2-7). Figs. (2 and 3) show the specific volumes for ferruginous soil, horizon A and ferralitic soil, horizon A, respectively, as representatives of the eight soils: the estimates of $\overline{V_{\mathrm{s}}}=\bar{V}$ (see Eq. (8)), ${\overline{V_{1}}}^{\prime}$ (Eq. (11)), and (corrected) $\bar{V}_{1}$ (Eq. (12) and (13)). In addition, crack volumes $\bar{V}_{\text {crl }}^{\prime}\left(\right.$ Eq. (5)) and (corrected) $\bar{V}_{\text {crl }}$ (Eq. (6)) are indicated by two-ended arrows. In Fig. (2 and 3) one can see the appreciable difference between the layer volume in Bronswijk's approximation $\left(\bar{V}_{1}^{\prime}\right)$, the corrected layer volume $\left(\overline{V_{1}}\right)$, and the soil matrix volume $(\bar{V})$.

Figs. (4 and 5), as examples, show the specific crack volume in the layer before $\left(\bar{V}_{\mathrm{crl}}{ }^{\prime}-\right.$ Bronswijk's approximation; Eq. (5)) and after ( $\bar{V}_{\mathrm{crl}}$; Eq. (6)) correction for ferruginous 


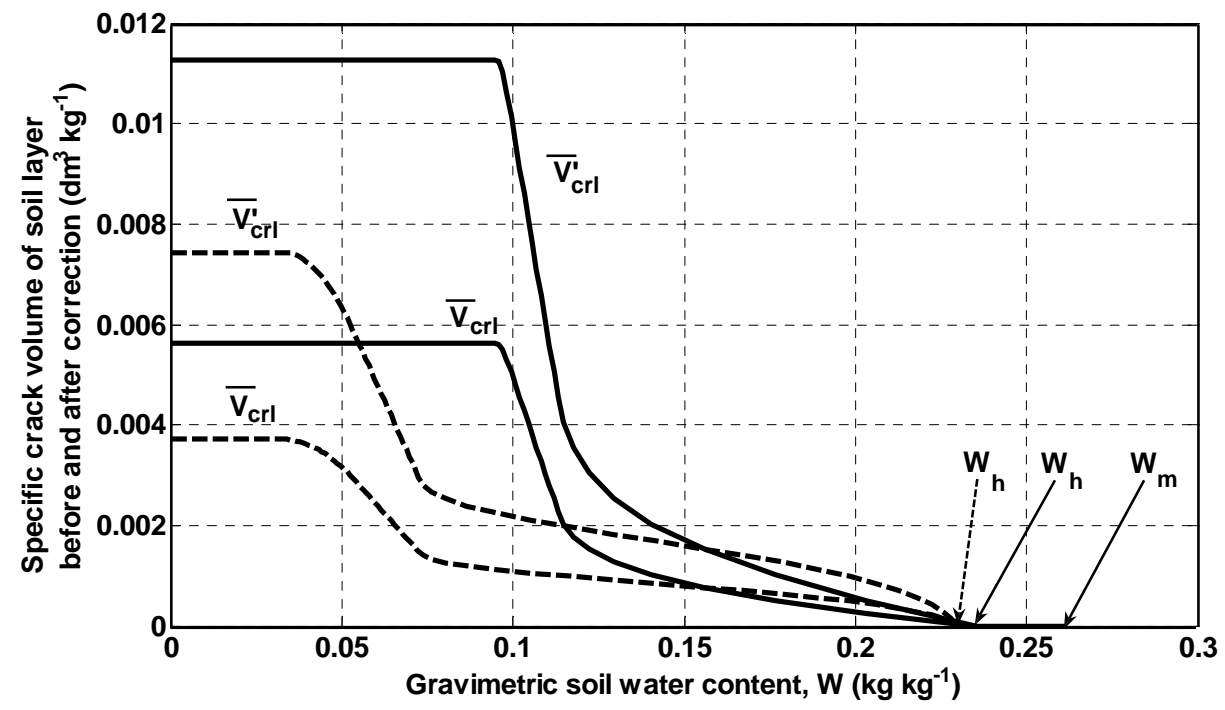

Fig. (4). Two examples of estimates, based on the reference shrinkage curve $[11,13]$, of the crack volume in the layer before $\left(\bar{V}_{\text {crl }}{ }^{\prime}(W)\right)$ and $\operatorname{after}\left(\bar{V}_{\mathrm{crl}}(W)\right)$ correction for ferruginous soil [18], horizon B1 (solid lines) and AB (dashed lines). $W_{\mathrm{h}}$ is the water content of starting shrinkage (the solid arrow - horizon $\mathrm{B} 1$; the dashed arrow - horizon $\mathrm{AB}$ ). $W_{\mathrm{m}}$ is the maximum water content of horizon $\mathrm{B} 1$.

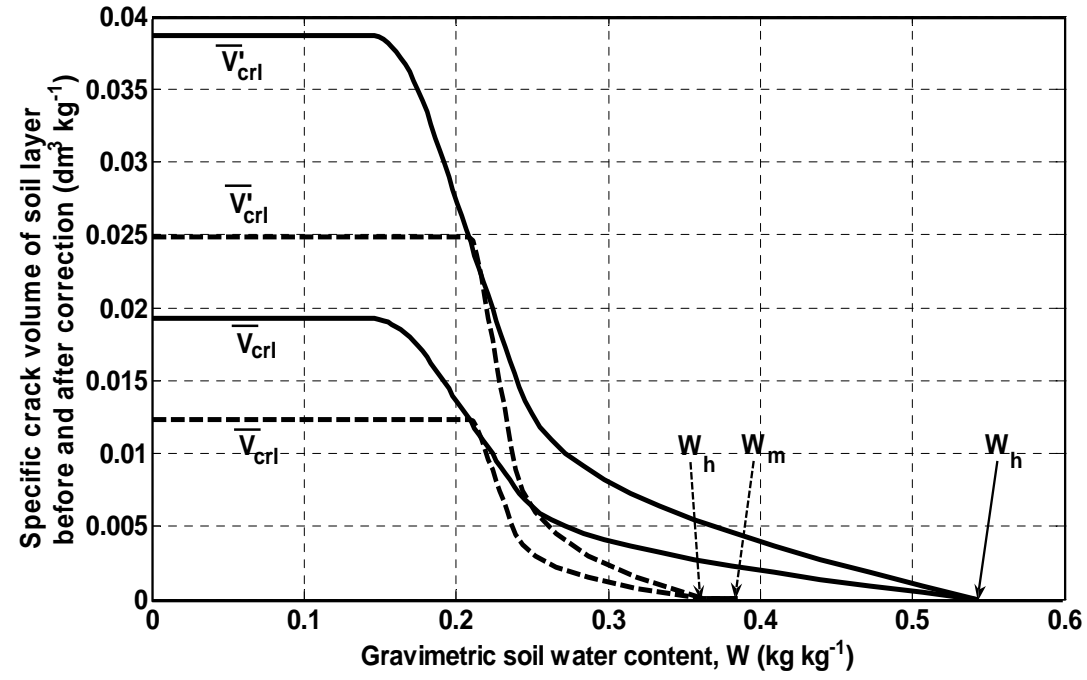

Fig. (5). Two examples of estimates, based on the reference shrinkage curve [11,13], of the crack volume in the layer before $\left(\bar{V}_{\text {crl }}{ }^{\prime}(W)\right)$ and after $\left(\bar{V}_{\mathrm{crl}}(W)\right)$ correction for ferralitic soil [18], horizon A (solid lines) and B2 (dashed lines). $W_{\mathrm{h}}$ is the water content of starting shrinkage (the solid arrow - horizon A; the dashed arrow - horizon B2). $W_{\mathrm{m}}$ is the maximum water content of horizon B2.

soil, horizons B1 and AB (Fig. 4) and for ferralitic soil, horizons A and B2 (Fig. 5). Note that in the case under consideration due to Eq. (7) and (8) the crack volume of cores, $\bar{V}_{\text {crs }}=0$. One can see that in all cases the corrected crack volume, $\bar{V}_{\mathrm{crl}}$ is approximately half as much compared to $\bar{V}_{\text {crl }}{ }^{\prime}$ in all the $0<W<W_{\mathrm{h}}$ range. Even at small crack volume as in the case of ferruginous soil, horizon A (see Fig. 2), when the maximum of $\bar{V}_{\text {crl }} \cong 1.1510^{-3} \mathrm{dm}^{3} \mathrm{~kg}^{-1}$ and $\bar{V}_{\text {crl }}{ }^{\prime} \cong 2.3$
$10^{-3} \mathrm{dm}^{3} \mathrm{~kg}^{-1}$, the difference between $\bar{V}_{\mathrm{crl}}{ }^{\prime}$ and $\bar{V}_{\mathrm{crl}}$ is crucially important for correct and dependable estimation of soil hydraulic properties.

It follows from Eq. (2), (3) and (8) as well as from the isotropy of the (core) matrix shrinkage that in the case under consideration

$r_{\mathrm{s}}^{\prime}(W)=r_{\mathrm{SM}}(W)=3$

Note that $r_{\mathrm{s}}^{\prime}$ gives the shrinkage geometry factor for both a soil layer and soil core in Bronswijk's approximation. Figs. 


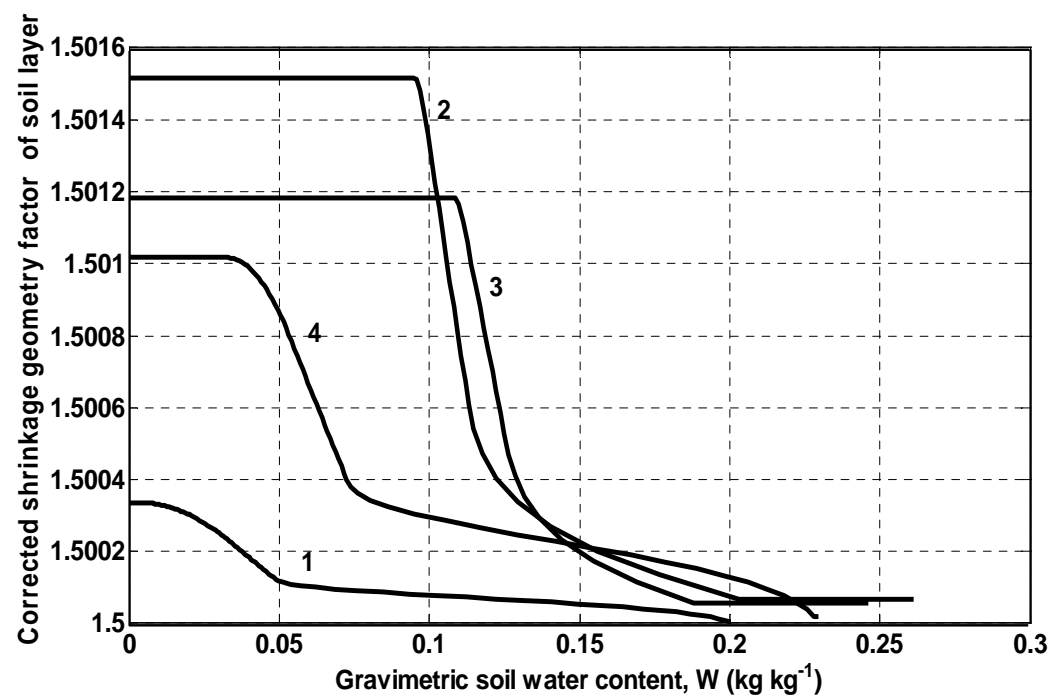

Fig. (6). Estimates of the corrected shrinkage geometry factor of a soil layer, $r_{\mathrm{s}}$ based on the reference shrinkage curves [11,13] for ferruginous soil [18]: 1-horizon A; 2-horizon B1; 3-horizon B2; 4-horizon AB.

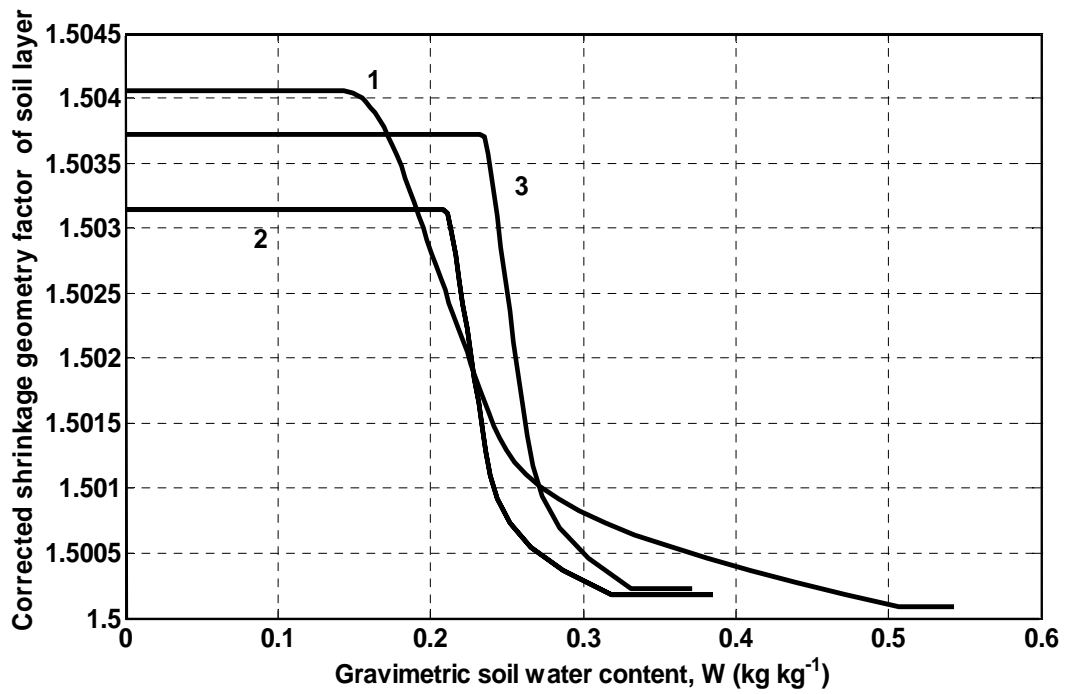

Fig. (7). Estimates of the corrected shrinkage geometry factor of a soil layer, $r_{\mathrm{s}}$ based on the reference shrinkage curves [11, 13] for a ferralitic soil [18]: 1-horizon A; 2-horizon B2; 3-horizon AB.

(6 and 7) show estimates of the corrected shrinkage geometry factor, $r_{\mathrm{s}}$ for the soil layer (Eq. (4)) of the four ferruginous soils and three ferralitic soils, respectively $\left(r_{\mathrm{s}}(W)\right.$ for horizons B1 and B2 of ferralitic soil were very close). One can see in Fig. (6 and 7) two major features of the corrected $r_{\mathrm{s}}$. The former one is the essential difference between $r_{\mathrm{s}}^{\prime}=3$ (Bronswijk's approximation) and corrected $r_{\mathrm{s}}$ for all eight soils. The latter is that the initial $r_{\mathrm{s}}$ value is equal to 1.5 and $r_{\mathrm{s}}$ grows with drying in a small range close to 1.5 . We will show that the latter feature is the direct consequence of isotropic core matrix shrinkage without cracking for the soils and core samples under consideration. In the case of core shrinkage without cracks and isotropic core matrix shrinkage (assumption of [18]), the specific volume of the soil matrix, $\bar{V}$ (the reference shrinkage curve) and core diameter, $d$ are connected as
$\bar{V}(W) / \overline{V_{\mathrm{h}}}=\left(d(W) / d_{\mathrm{h}}\right)^{3}$

By definition, the current specific layer volume in Bronswijk's approximation, ${\overline{V_{1}}}_{1}^{\prime}$ is connected with the current core diameter, $d$ (or height $h$, cf. Eq. (11)) as

${\overline{V_{1}}}^{\prime}(W) / \overline{V_{\mathrm{h}}}=d(W) / d_{\mathrm{h}}$

By definition of the corrected shrinkage geometry factor of soil layer, $r_{\mathrm{s}}(W)$, one can write (cf. Eq. (4))

$\bar{V}(W) / \bar{V}_{\mathrm{h}}=\left(\bar{V}_{1}(W) / \bar{V}_{\mathrm{h}}\right)^{r}(W)$ 
where $\bar{V}_{1}$ is the corrected specific volume of a soil layer with cracks. Finally, $\overline{V_{1}}$ and ${\overline{V_{1}}}^{\prime}$ in the case of core shrinkage without cracks and isotropic core matrix shrinkage, are connected as (cf. Eq. (12) and (13))

$\bar{V}_{1}(W)={\overline{V_{1}}}^{\prime}(W)\left(1+d(W) / d_{\mathrm{h}}\right)^{2} / 4$

Combining Eq. (15)-(18) one obtains the following relation

$$
\begin{aligned}
& \left({\overline{V_{1}}}^{\prime}(W) / \overline{V_{\mathrm{h}}}\right)^{3}=\left({\overline{V_{1}}}^{\prime}(W) / \overline{V_{\mathrm{h}}}\right)^{r(W)} \\
& \cdot\left(1+{\overline{V_{1}}}^{\prime}(W) / \overline{V_{\mathrm{h}}}\right)^{2 r}(W) \cdot 4_{\mathrm{s}}^{-r(W)}
\end{aligned}
$$

Denoting in Eq. (19) $\bar{V}_{1}^{\prime}(W) / \bar{V}_{\mathrm{h}} \equiv(1-x)$ one can rewrite Eq. (19) as

$(1-x)^{3}=(1-x)^{r} \cdot(2-x)^{2 r} \mathrm{~s} \cdot 4^{-r} \mathrm{~s}$

At the shrinkage start $W \rightarrow W_{\mathrm{h}}$ (see Fig. 2 and 3), $r_{\mathrm{s}}(W) \rightarrow$ $r_{\mathrm{s}}\left(W_{\mathrm{h}}\right) \equiv r_{\mathrm{sh}}, \quad \bar{V}_{1}^{\prime}(W) \rightarrow \bar{V}_{\mathrm{h}} \quad$ (see $\quad$ Fig. $\quad 2 \quad$ and $\left.\quad \mathbf{3}\right)$, then $x \equiv 1-{\overline{V_{1}}}^{\prime}(W) / \overline{V_{\mathrm{h}}} \rightarrow 0$, and Eq. (20) gives

$1-3 x \cong\left(1-r_{\mathrm{sh}} x\right)^{2} \cong 1-2 r_{\mathrm{sh}} x$

That is, the corrected shrinkage geometry factor of a soil layer at the shrinkage start, $r_{\mathrm{sh}} \cong 1.5$.

It is worth emphasizing that the above results reflect the specifics of data from [18] (isotropic shrinkage of core matrix without cracks at any water content). In general, $\bar{V}_{\mathrm{S}}(W)>\bar{V}(W)$ unlike in Fig. (2 and $\left.\mathbf{3}\right)$ at the expense of core cracks (see Fig. 1). Moreover, in the general case $r_{\mathrm{s}}^{\prime}(W) \neq r_{\mathrm{SM}}(W) \neq 3$ unlike in Eq. (14). In addition, in the general case the corrected $r_{\mathrm{s}}(W)$ dependence, unlike in Fig. 6 and 7, can have the maximum with a water content decrease and can start from a value that differs from 1.5 (see Fig. (8) from [7]). However, we aimed at illustrating how the reference shrinkage curve of a soil can be used to estimate the corrected values of the shrinkage geometry factor and crack volume at soil shrinkage. The above results give corresponding examples and clearly show the essential differences be-

tween $\bar{V}_{1}^{\prime}, \bar{V}_{1}$, and $\bar{V}$ (Fig. $\mathbf{2}$ and $\mathbf{3}$ ), $\bar{V}_{\text {crl }}$ and $\bar{V}_{\text {crl }}{ }^{\prime}$ (Fig. 4 and 5) as well as between the $r_{\mathrm{s}}(W)$ dependencies in Fig. 6 and 7 and $r_{\mathrm{s}}^{\prime}(W)=r_{\mathrm{SM}}(W)=3$ (Eq. (14)). These differences, in turn, indicate the necessity to introduce corrections into $r_{\mathrm{s}}$ values and soil crack volume estimates from Bronswijk's approximation.

\section{CONCLUSION}

In addition to $[6,7]$ this work argues and illustrates the necessity of introducing corrections into the shrinkage geometry factor and crack volume compared to values that are determined from Bronswijk's approximation. In line with that the work illustrates the use and role of the reference shrinkage curve [11-13] for obtaining the corrected values of the shrinkage geometry factor and soil crack volume. We believe that this promising application of the soil reference shrinkage curve will be expanded with the accumulation of corresponding data.

\section{REFERENCES}

[1] Bronswijk JJB. Modeling of water balance, cracking and subsidence of clay soils. J Hydrol 1988; 97: 199-212.

[2] Bronswijk JJB. Prediction of actual cracking and subsidence in clay soils. Soil Sci 1989; 148: 87-93.

[3] Bronswijk JJB. Relation between vertical soil movements and water-content changes in cracking clays. Soil Sci Soc Am J 1991; 55: 1220-1226.

[4] Bronswijk JJB. Drying, cracking, and subsidence of a clay soil in a lysimeter. Soil Sci 1991; 152(2): 92-99.

[5] Bronswijk JJB. Shrinkage geometry of a heavy clay soil at various stresses. Soil Sci Soc Am J 1990; 54: 1500-1502.

[6] Chertkov VY, Ravina I, Zadoenko V. An approach for estimating the shrinkage geometry factor at a moisture content. Soil Sci Soc Am J 2004; 68: 1807-1817.

[7] Chertkov VY. The shrinkage geometry factor of a soil layer. Soil Sci Soc Am J 2005; 69: 1671-1683.

[8] Hallaire V. Evolution of crack networks during shrinkage of a clay soil under grass and winter wheat crops: in ISSS Symp. on water and solute movement in heavy clay soils ILRI, Conference Proceedings, Wageningen, The Netherlands, Eds. Bouma J, Raats PAC. 1984; 49-53.

[9] Chertkov VY. Modeling the pore structure and shrinkage curve of soil clay matrix. Geoderma 2000; 95: 215-246.

[10] Chertkov VY. Modelling the shrinkage curve of soil clay pastes. Geoderma 2003; 112: 71-95.

[11] Chertkov VY. The reference shrinkage curve at higher than critical soil clay content. Soil Sci Soc Am J 2007; 71(3): 641-655.

[12] Chertkov VY. The reference shrinkage curve of clay soil. Theor and Appl Fracture Mechanics 2007; 48(1): 50-67.

[13] Chertkov VY. The soil reference shrinkage curve. Open Hydrol J 2007; 1: 1-18.

[14] Garnier P, Rieu M, Boivin P, Vauclin M, Baveye P. Determining the hydraulic properties of a swelling soil from a transient evaporation experiment. Soil Sci Soc Am J 1997; 61(6): 1555-1563.

[15] Crescimanno G, Provenzano G. Soil shrinkage characteristic curve in clay soils: Measurement and prediction. Soil Sci Soc Am J 1999; 63: 25-32.

[16] Cornelis WM, Corluy J, Medina H, Hartmann R, Van Meirvenne M, Ruiz ME. A simplified parametric model to describe the magnitude and geometry of soil shrinkage. Eur J Soil Sci 2006; 57: 258268.

[17] Peng X, Horn R. Anisotropic shrinkage and swelling of some organic and inorganic soils. Eur J Soil Sci 2007; 58: 98-107.

[18] Braudeau E, Sene M, Mohtar RH. Hydrostructural characteristics of two African tropical soils. Eur J Soil Sci 2005; 56: 375-388.

(C) V.Y. Chertkov; Licensee Bentham Open.

This is an open access article licensed under the terms of the Creative Commons Attribution Non-Commercial License (http://creativecommons.org/licenses/by-nc/3.0/) which permits unrestricted, non-commercial use, distribution and reproduction in any medium, provided the work is properly cited. 